\title{
Relationship between the Craniovertebral Angle, Cervical Lordosis, and Cervical Muscles
}

\author{
Joo-Hee Park, PT, Ph.D · One-Bin Lim, PT, Ph. 1 $^{\dagger \dagger}$ \\ Department of Physical Therapy, College of Software and Digital Healthcare Convergence, Yonsei University \\ ${ }^{1}$ Department of Physical Therapy, Mokpo Science University
}

Received: December 22021 / Revised: December 62021 / Accepted: December 302021

(C) 2022 J Korean Soc Phys Med

\begin{abstract}
| Abstract |
PURPOSE: This study examined the relationship between the cervical lordosis angle (CLA) and the craniovertebral angle (CVA) and between CVA and a cross-sectional area (CSA) of the cervical muscles.
\end{abstract}

METHODS: In 17 healthy college students, the CLA was measured using a posterior tangent technique. The CVA was analyzed using photographic images, and the CSA of the cervical muscles, including longus colli, longus capitis, and sternocleidomastoid, was evaluated using computerized tomography. The Pearson's correlation coefficient was used to determine the relationship between these variables and a neck disability index.

RESULTS: The CVA correlated with the CLA and with a CSA of longus colli $(\mathrm{r}=.487, \mathrm{p}=.047$ and $\mathrm{r}=.592, \mathrm{p}=.012$, respectively). The CLA correlated with a CSA of longus colli $(\mathrm{r}=.578, \mathrm{p}=.015)$.

CONCLUSION: This study clarified the relationship between the postural, structural, and muscular changes in the cervical regions. A significant correlation was observed

$\dagger$ Corresponding Author: One-Bin Lim onebin007@naver.com, https://orcid.org/0000-0002-5281-3463

This is an Open Access article distributed under the terms of the Creative Commons Attribution Non-Commercial License (http://creativecommons.org/licenses/by-nc/3.0) which permits unrestricted non-commercial use, distribution, and reproduction in any medium, provided the original work is properly cited. between the CVA and a CSA of the longus colli with the CLA and between the CVA and the CSA of the longus colli. Prescription strengthening exercises for the deep neck flexors for individuals with a forward head posture and reduced cervical lordosis are useful.

Key Words: Exercise, Lordosis, Muscles, Neck

\section{Introduction}

Forward head posture (FHP) has been defined as the forward translation of the cervical vertebrae, inducing flexion of the lower cervical spine and extension of the upper cervical spine [1]. These postural changes can affect the length-tension relationship of cervical musculature and increase the flexion moment in specific muscles. Therefore, habitual and prolonged FHP results in muscle imbalance as the body adapts to find efficient ways of holding the head up for straight-ahead vision [2]. Muscle imbalance in FHP shows a tendency wherein deep neck flexor muscles, such as longus colli, become elongated and weakened. In contrast, anterior neck muscles (sternocleidomastoid and scalenus anterior) and capital extensor muscles (semispinalis capitis) become shortened and tightened [1].

The reduced cervical spine curve is also related to a muscle imbalance. Yoon et al. [3] reported a positive 
correlation between the ratio of cervical flexor and extensor muscles and cervical lordosis. They suggested that restoring cervical muscle balance is an effective way to maintain cervical lordotic curvature. In particular, weakness of the deep neck flexors reduces the ability to maintain the upright posture of the cervical spine because the deep neck flexors are important in postural support in the cervical region [4]. Therefore, strengthening the longus colli muscle increases the cervical lordotic curve [3-5].

Both FHP and a reduced cervical lordosis are related to muscle imbalance wherein deep neck flexors tend to become weak. Therefore, some studies have suggested that prolonged FHP can induce muscle imbalance that eventually leads to structural changes (reduced cervical lordosis), which, when occurring within the cervical spine, result in various clinical symptoms, such as neck pain [6] and myelopathy [7-9]. In this regard, several studies and websites have used the terms 'FHP' and 'reduced cervical curve' interchangeably, often placing 'cervical lordosis' in parentheses alongside to appear synonymous with FHP $[3,10]$.

Sun et al. (2014) [11] reported only a limited relationship between the FHP and cervical lordosis $(r=.2-3)$. Furthermore, Mayoux-Benhamou et al. [12] reported no relationship between the FHP and a cross-sectional area (CSA) of the longus colli using computerized tomography (CT). These findings concerning either a limited or a non-significant relationship can be challenged because they may have been influenced through using inappropriate variables to evaluate the FHP [11,12]. In previous studies, FHP was measured as the length of head protraction [11, 12]. Therefore, as the maximum torque value is usually normalized to the body mass index (BMI) value when making comparisons between individuals, length variables should be normalized according to the height and neck length to analyze this relationship.

There is general agreement that prolonged FHP induces muscle imbalances, changes cervical spine structure, and can lead to severe neck pain. On the other hand, previous studies have not substantiated this general agreement and have reported findings of FHP having little or no relationship with the cervical curve and cervical muscles $[3,12]$. Length measurements, which have been used to measure the amount of head protraction, have been imprecise when analyzing this relationship because the length variable includes information on the amount of head protraction along with height and neck length. Therefore, this relationship needs to be re-analyzed using angular measures, such as the CVA, to have more accurate, targeted measurements for clinicians and researchers [13]. The CVA has been verified for its reliability and validity $[14,15]$. As length may be a confounding variable, a CVA measurement for FHP would be more likely to provide an accurate result in terms of this relationship. Therefore, this study examined the relationship between the cervical lordosis and a CVA and between the CVA and CSA of cervical muscles using $\mathrm{CT}$ to measure these angles.

\section{Methods}

\section{Participants}

This study was conducted at the Yonsei University Mirae Campus in South Korea. This study comprised 17 asymptomatic student participants (age, 20-29 years) with no previous pathological or surgical history to limit the effects of age-related changes to cervical structures. The participants were excluded for the following reasons: if they had undergone a previous cervical operation; had diseases that could affect the cervical spine posture, such as cervical spine fracture, myelopathy, spinal cord tumor, and ossification of the posterior longitudinal ligament; inflammatory rheumatic diseases, such as ankylosing spondylitis and rheumatoid arthritis; had insufficient radiological or medical records. All participants volunteered for this study and were informed of the study procedures prior to participation. 


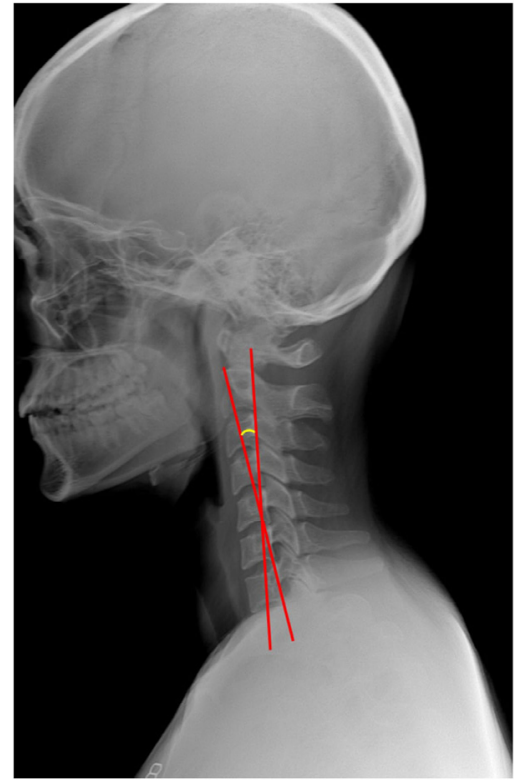

Fig. 1. Cervical Lordosis using the harrison posterior tangent technique.

\section{Measuring the cervical lordosis angle using} lateral X-ray images

Before X-ray scanning, the participants were requested to stand and look straight ahead, in a relaxed stance and in a neutral pelvic position, to prevent the effects of the pelvic tilt on cervical lordosis [16]. The cervical lordosis angle (CLA) was measured to represent the degree of cervical lordosis. This angular measurement between two lines parallel to the posterior surface of $\mathrm{C} 2$ and $\mathrm{C} 7$ is known as the Harrison posterior tangent technique [17] (Fig. 1). A professional radiologist measured x-ray data, and CLA was analyzed by one of the authors, PJH. A lower CLA indicates less cervical lordosis, indicating a straighter cervical curve. The CLA was reported to be a reliable measurement method, with good intra- and inter-observer reliability and small error ranges $[18,19]$.

\section{Cross-sectional areas (CSA) of cervical muscles}

The CSA of cervical muscles was measured. Each participant was requested to lie supine with their head positioned on a resting splint to prevent head rotation. The subjects were asked not to swallow or breathe. CT (Philips MX 16 slice CT scanner, Philips, Netherlands) images were used to measure the CSA of the longus colli, longus capitis, and sternocleidomastoid (SCM) muscles. Each muscle was scanned transversely, at a slice thickness of $5 \mathrm{~mm}$, at seven different cervical levels (C0-1, C1-2, C2-3, C3-4, C4-5, C5-6, and C6-7). The CSAs of longus colli, longus capitis, and SCM were assessed at the section plane of the $\mathrm{C6}^{-7}$, C1-2, and $\mathrm{C} 4-5$ vertebrae, respectively, because, at these levels, the central muscle fibers run from the highest origin point to the lowest insertion point and provide the clearest visualization of each muscle. The contours of each muscle were traced manually and measured three times to calculate the CSA using Image J software (National Institutes of Health, Bethesda, MD, USA). The data were averaged in the analysis (Fig. 2). A professional radiologist measured the CT data, and the CSA of each muscle was analyzed by one of the authors, LOB.

\section{The CVA using lateral photographic images}

The CVA was recorded using photographic images taken with a participant in their usual standing posture, with a
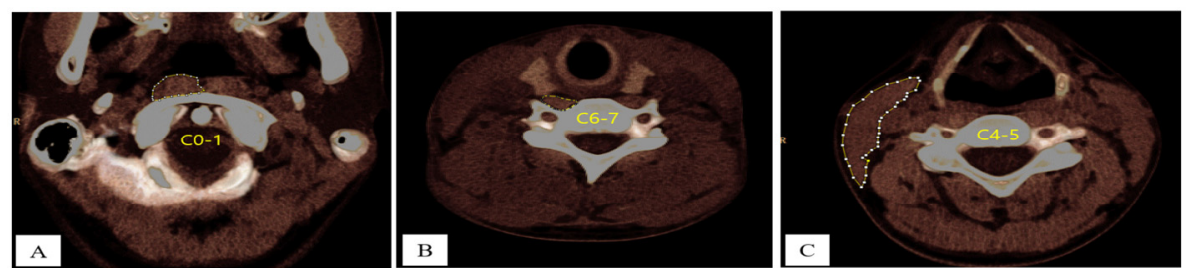

Fig. 2. Cross-sectional areas (CSA) of cervical muscles on CT. (A) C1-2 level, showing the longus capitis muscle. (B) C6-7 level, showing the longus colli muscle.

(C) C4-5 level, showing the sternocleidomastoid muscle. 


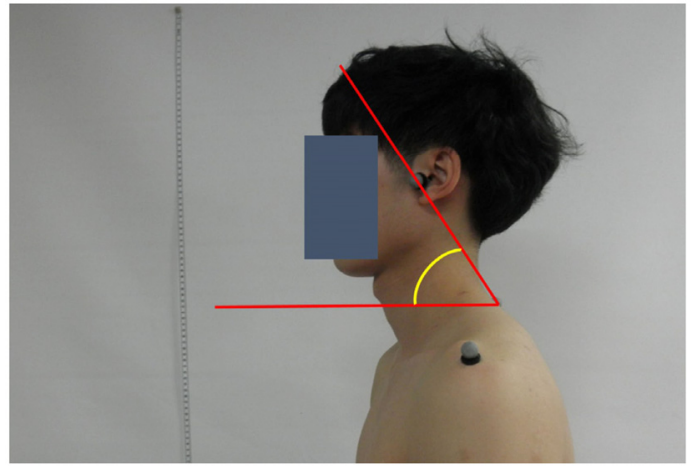

Fig. 3. Craniovertebral angle (CVA).

neutral pelvic tilt, which was a similar posture to that used to obtain plain cervical radiographic images. One experienced physical therapist, $\mathrm{PJH}$, located and marked the spinous process of the $\mathrm{C} 7$ vertebrae and the tragus, and the CVA was analyzed by one of the authors, LOB. The CVA comprised the angle between a horizontal line passing through $\mathrm{C} 7$ and a line extending from the tragus to $\mathrm{C} 7$, which was quantified using Image $\mathrm{J}$ software (National Institutes of Health, Bethesda, MD, USA). A lower CVA represented a greater FHP (Fig. 3).

\section{The neck disability index}

The Korean version of the neck disability index (NDI) $(\mathrm{ICC}=.927$, Cronbach's alpha $=.82)$ was used to assess the neck disability [20]. The NDI involves using a questionnaire to determine how neck pain influences daily life and has a total score of 50 points. A high score represents severe neck disability.

\section{Risk of bias}

Different assessors conducted each measure, with no information provided concerning the participants' status or the experimental purpose of the study, to prevent potential exposure of information regarding the study findings. All data were blinded during the study, and the researchers only provided their results after all the measurements had been taken.
Table 1. Participants' Demographic Characteristics $(\mathrm{N}=17)$

\begin{tabular}{cc}
\hline Characteristics & Value \\
\hline Age (years) & $22.35 \pm 1.80$ \\
Sex & $10 / 7$ \\
(male / female) & $50.27 \pm 7.18$ \\
Craniovertebral angle & \\
(CVA, degrees) & $9.74 \pm 8.07$ \\
Cervical lordosis angle & \\
(CLA, degrees) & \\
CLA according to gender & $11.68 \pm 9.60 / 9.46 \pm 5.94$ \\
(male / female) & $.11 \pm .07$ \\
CSA of longus colli & $1.23 \pm .38$ \\
CSA of longus capitis & $4.00 \pm 1.41$ \\
CSA of SCM & $11.88 \pm 13.00$ \\
\hline NDI &
\end{tabular}

Values are given as mean \pm standard deviation; CLA: cervical lordosis angle; CSA: cross-sectional area; CVA: craniovertebral angle; NDI: neck disability index; SCM: sternocleidomastoid

\section{Statistical analysis}

The Pearson's correlation coefficient was used to investigate the relationship between the CVA, the CLA, CSA of the cervical muscles (longus colli, longus capitis, and SCM), and the NDI. All analyses were conducted using SPSS, version 25 (SPSS Inc., Chicago, IL, USA) statistical software. The p-value was set to .05 , and $95 \%$ confidence intervals were assumed for all analyses.

\section{Results}

Table 1 lists the participants' demographic characteristics. Seventeen participants (10 males, 7 females) were included in this study, and the mean age was $22.35 \pm 1.80$ years. The average CVA was $50.27 \pm 7.18$ degrees, and the average CLA was $9.74 \pm 8.07$ degrees.

Table 2 lists the Pearson's correlation coefficients concerning the CVA, CLA, and CSA of longus colli, longus capitis, and SCM, as well as the NDI. The CVA correlated with the CLA and the CSA of longus colli $(r=.487, p$ 
Table 2. Pearson's Correlation Coefficient Results

\begin{tabular}{|c|c|c|c|c|c|c|}
\hline & CLA & CVA & CSA of longus colli & CSA of longus capitis & CSA of SCM & NDI \\
\hline CLA & 1.000 & & & & & \\
\hline CVA & $\begin{array}{c}.487^{*} \\
(\mathrm{p}=.047)\end{array}$ & 1.000 & & & & \\
\hline CSA of longus colli & $\begin{array}{c}.578^{*} \\
(p=.015)\end{array}$ & $\begin{array}{c}.592^{*} \\
(\mathrm{p}=.012)\end{array}$ & 1.000 & & & \\
\hline CSA of longus capitis & $\begin{array}{c}.141 \\
(\mathrm{p}=.588)\end{array}$ & $\begin{array}{c}.124 \\
(p=.634)\end{array}$ & $\begin{array}{c}.400 \\
(\mathrm{p}=.112)\end{array}$ & 1.000 & & \\
\hline CSA of SCM & $\begin{array}{c}-.053 \\
(\mathrm{p}=.839)\end{array}$ & $\begin{array}{c}.127 \\
(p=.626)\end{array}$ & $\begin{array}{c}.104 \\
(p=.691)\end{array}$ & $\begin{array}{c}.658^{*} \\
(\mathrm{p}=.004)\end{array}$ & 1.000 & \\
\hline NDI & $\begin{array}{c}-.178 \\
(\mathrm{p}=.495)\end{array}$ & $\begin{array}{c}.337 \\
(\mathrm{p}=.185)\end{array}$ & $\begin{array}{c}.264 \\
(p=.305)\end{array}$ & $\begin{array}{c}.184 \\
(p=.480)\end{array}$ & $\begin{array}{c}-.073 \\
(\mathrm{p}=.781)\end{array}$ & 1.000 \\
\hline
\end{tabular}

$=.047$ and $\mathrm{r}=.592, \mathrm{p}=.012$, respectively). The CLA correlated with the CSA of the longus colli $(r=.578, \mathrm{p}$ $=.015)$. On the other hand, the NDI did not show a significant correlation with any other parameter.

\section{Discussion}

The angular variable is used widely to assess FHP in studies and in clinical settings. Among many angular variables, the craniovertebral angle (CVA) is considered a good indicator to differentiate between the normal head posture and FHP. Therefore, it is necessary to re-analyze this relationship using angular measures, such as the CVA, to provide more accurate, targeted measurements for clinicians and researchers.

These findings suggest that there was a statistically significant but fair relationship between the FHP and cervical lordosis $(\mathrm{r} \leq .25$, little or no relationship; .25 $<\mathrm{r}<.5$, low to fair relationship; $.5<\mathrm{r} .75$, moderate to good, and; $r \geq .75$, a strong relationship) [23]. The results showed a stronger relationship than those studies reporting a limited or non-significant relationship between the length of head protraction and cervical lordosis $[3,12]$.
Furthermore, a significant and moderate relationship was observed between a CSA of the longus colli and cervical lordosis, whereas an early study reported no significant relationship [12]. The use of length as a variable may identify relationships less accurately and on a more limited scale between the variables compared to using angular variables. This is because the length variable is more likely to be a confounding factor in any relationship [23].

The moderate relationship between FHP and longus colli suggested that a higher FHP indicated a weaker longus colli muscle. The longus colli contains a high density of muscle spindles and proprioception receptors. Therefore, longus colli weakness reduces the stability of the head and neck and limits neck motion [24,25]. Furthermore, longus colli plays an important role in holding spinal lordosis against the forces of gravity and the weight of the head. A weak longus colli muscle cannot support the head effectively, thereby placing stress on structures located in the cervical spine, which can interfere with the upright stability. A previous study reported that craniocervical flexion exercise targeted at retraining longus colli improves the cervical lordosis and FHP and even reduces neck pain [4]. This finding supports the results of previous meta-analyses 
studies, in which longus colli strengthening exercise was found to improve the overall neck problems [26].

Other cervical muscles, such as SCM and longus capitis, did not correlate with any other parameters, including FHP. Consistent with these study findings, Yoon et al. (2018) [3] reported that the SCM and longus capitis did not correlate with cervical lordosis. Neck disability did not correlate with any other parameters. This study hypothesized that neck disability increased together with increasing FHP and decreasing cervical lordosis. The study participants were relatively young, and greater head protraction or cervical hypolordosis did not result in severe disability.

Approximately $50 \%$ of healthy asymptomatic college students have postural and structural changes to the cervical spine $[21,22,27]$. The average CVA and CLA of the 17 participants were $50.27 \pm 7.18$ degrees and $9.74 \pm 8.07$ degrees, respectively. These CVA and CLA results appeared to be high, considering that the average CLA of 20-29-year-old participants was approximately 20 degrees and that a CLA $<20$ degrees resulted in a two-fold risk of cervicogenic symptoms [21]. A sedentary lifestyle and overuse of smartphones are likely to have influenced these results. Moreover, maintaining an upright posture and performing exercises have previously been recommended for neck health [28].

This study found that a weaker longus colli muscle indicated a greater FHP and reduced cervical lordosis. Thus, this study provided supporting evidence in favor of prescribing strengthening exercises of deep neck flexors for people with FHP and reduced cervical lordosis. Mayoux-Benhamou et al. (1994) [12] found a negative relationship between cervical lordosis and longus colli. They reported that cervical lordosis is increased markedly if the longus colli muscle becomes weak. This contrasting result might be due to a relatively large number of subjects with FHP and reduced cervical lordosis. As mentioned above, all the subjects in the present study had reduced cervical lordotic curve, but Mayoux-Benhamou et al. (1994) reported that only four subjects (11\%) showed reduced cervical lordosis [12]. Future studies will need to compare the correlation of the cervical muscle with the reduced cervical lordosis.

A previous meta-analysis showed found that the cervical lordosis was higher in males than in females, but there was no relationship with symptoms [29]. This study found a difference in the lordotic curve according to gender. On the other hand, there was no correlation between CLA and CSA of each muscle according to gender. Nevertheless, the number of subjects of this study was insufficient (10 males and 7 females). Therefore, a future study needs to analyze the relationship according to gender.

\section{Conclusion}

A relationship was observed between the neck posture, spine structure, and cervical muscles, but research concerning the precise relationships between these variables showed only a limited relationship or no statistical significance. On the other hand, using length as a variable may be a confounding factor influencing the results of studies assessing these relationships. Therefore, this study used the CVA to measure the FHP to analyze these relationships and reported a fair relationship between cervical lordosis and neck posture and a moderate relationship between the CSA of the longus colli and cervical lordosis. This study provides further evidence to support the effects of strengthening exercises for deep cervical flexor muscles to enhance posture and cervical lordosis.

\section{Acknowledgments}

The authors would like to thank the participants who volunteered for this study. 


\section{References}

[1] Neumann DA. Kinesiology of the Musculoskeletal System: Foundations for Rehabilitation (2nd ed). St Louis. Mosby. 2009.

[2] Lindfors P, Von Thiele U, Lundberg U. Work characteristics and upper extremity disorders in female dental health workers. J Occup Health. 2006;48(3):192-7.

[3] Yoon SY, Moon HI, Lee SC, et al. Association between cervical lordotic curvature and cervical muscle crosssectional area in patients with loss of cervical lordosis. Clin Anat. 2018;31(5):710-5.

[4] Chung S, Jeong YG. Effects of the craniocervical flexion and isometric neck exercise compared in patients with chronic neck pain: a randomized controlled trial. Physiother Theory Pract. 2018;34(12):916-25.

[5] Sheikhhoseini R, Shahrbanian S, Sayyadi P, et al. Effectiveness of therapeutic exercise on forward head posture: a systematic review and meta-analysis. J Manipulative Physiol Ther. 2018;41(6):530-9.

[6] Harrison DD, Harrison DE, Janik TJ, et al. Modeling of the sagittal cervical spine as a method to discriminate hypolordosis: results of elliptical and circular modeling in 72 asymptomatic subjects, 52 acute neck pain subjects, and 70 chronic neck pain subjects. Spine. 2004;29(22): 2485-92.

[7] Alpayci M, Ilter S. Isometric exercise for the cervical extensors can help restore physiological lordosis and reduce neck pain: a randomized controlled trial. Am J Phys Med Rehabil. 2017;96(9):621-6.

[8] Fujimoto Y, Oka S, Tanaka N, et al. Pathophysiology and treatment for cervical flexion myelopathy. Eur Spine J. 2002;11(3):276-85.

[9] Shimizu K, Nakamura M, Nishikawa Y, et al. Spinal kyphosis causes demyelination and neuronal loss in the spinal cord: a new model of kyphotic deformity using juvenile Japanese small game fowls. Spine. 2005;30(21): 2388-92.
[10] Falla D, Jull G, Russell T, et al. Effect of neck exercise on sitting posture in patients with chronic neck pain. Phys Ther. 2007;87(4):408-17.

[11] Sun A, Yeo HG, Kim TU, et al. Radiologic assessment of forward head posture and its relation to myofascial pain syndrome. Ann Rehabil Med. 2014;38(6):821-6.

[12] Mayoux-Benhamou MA, Revel M, Vallée C, et al. Longus colli has a postural function on cervical curvature. Surg Radiol Anat. 1994;16(4):367-71.

[13] Lee HS, Chung HK, Park SW. The Analysis of severity of forward head posture with observation and photographic method. J Korean Soc Phys Med. 2015;10(3):9-17.

[14] Gadotti IC, Biasotto-Gonzalez DA. Sensitivity of clinical assessments of sagittal head posture. J Eval Clin Pract. 2010;16(1):141-4.

[15] Gadotti IC, Magee DJ. Validity of surface measurements to assess craniocervical posture in the sagittal plane: a critical review. Physical Therapy Reviews. 2008;13(4): $258-68$.

[16] Scheer JK, Tang JA, Smith JS, et al. Cervical spine alignment, sagittal deformity, and clinical implications: a review. J Neurosurg Spine. 2013;19(2):141-59.

[17] Grob D, Frauenfelder H, Mannion AF. The association between cervical spine curvature and neck pain. Eur Spine J. 2007;16(5):669-78.

[18] Harrison DE, Harrison DD, Cailliet R, et al. Cobb method or harrison posterior tangent method: which to choose for lateral cervical radiographic analysis. Spine. 2000;25(16):2072-8.

[19] Harrison DE, Cailliet R, Harrison DD, et al. Reliability of centroid, Cobb, and Harrison posterior tangent methods: which to choose for analysis of thoracic kyphosis. Spine. 2001;26(11):E227-34.

[20] Song KJ, Choi BW, Kim SJ, et al. Cross-cultural adaptation and validation of the korean version of the neck disability index. J Korean Orthop Assoc. 2009;44(3):350-9.

[21] McAviney J, Schulz D, Bock R, et al. Determining the relationship between cervical lordosis and neck complaints. 
J Manipulative Physiol Ther. 2005;28(3):187-93.

[22] Ruivo RM, Pezarat-Correia P, Carita AI. Effects of a resistance and stretching training program on forward head and protracted shoulder posture in adolescents. J Manipulative Physiol Ther. 2017;40(1):1-10.

[23] Portney LG. Foundations of Clinical Research: Applications to Evidence-based Practice (4th ed). Philadelphia. FA Davis. 2020.

[24] Armstrong BS, McNair PJ, Williams M. Head and neck position sense in whiplash patients and healthy individuals and the effect of the cranio-cervical flexion action. Clin Biomech. 2005;20(7):675-84.

[25] Röijezon U, Björklund M, Bergenheim M, et al. A novel method for neck coordination exercise - a pilot study on persons with chronic non-specific neck pain. J Neuroeng Rehabil. 2008;5(1):1-10.

[26] Park JH, Jeon HS, Kim JH, et al. Effects of biofeedback based deep neck flexion exercise on neck pain: meta-analysis. Phys Ther Korea. 2021;28(1):18-26.

[27] Yochum TR, Rowe LJ. Essentials of Skeletal Radiology. Philadelphia. Williams and Wilkens. 1987.

[28] Park JH, Kang SY, Lee SG, et al. The effects of smart phone gaming duration on muscle activation and spinal posture: pilot study. Physiother Theory Pract. 2017;33(8): 661-9.

[29] Guo GM, Li J, Diao QX, et al. Cervical lordosis in asymptomatic individuals: a meta-analysis. J Orthop Surg Res. 2018;13(1):147. 